\title{
DNA methylation-mediated Klotho silencing is an independent prognostic biomarker of head and neck squamous carcinoma
}

This article was published in the following Dove Medical Press journal: Cancer Management and Research

\author{
Yun Zhu' \\ Xuehong $\mathrm{CaO}^{2}$ \\ Xiaomeng Zhang' \\ Quan Chen ${ }^{3}$ \\ Lei Wen ${ }^{2}$ \\ Ping Wang ${ }^{4}$ \\ 'Department of Otorhinolaryngology, \\ Union Hospital, Tongji Medical \\ College, Huazhong University of \\ Science and Technology, Wuhan, \\ Hubei 430022, China; ${ }^{2}$ Department \\ of Traditional Chinese Medicine, \\ Medical College, Xiamen University, \\ Xiamen, Fujian Province 36II02, \\ China; ${ }^{3}$ Department of Radiology, \\ Union Hospital, Tongji Medical \\ College, Huazhong University of \\ Science and Technology, Wuhan, \\ Hubei 430022, China; ${ }^{4}$ Department \\ of Pediatrics, Union Hospital, Tongji \\ Medical College, Huazhong University \\ of Science and Technology, Wuhan \\ 430022, China
}

Purpose: To study the prognostic value of klotho (KL) and its promoter DNA methylation in head and neck squamous cell carcinoma (HNSCC) and to assess their associations with the autophagy gene LC3 and the RNA transferase gene NSUN2.

Materials and methods: Upper quartile normalized RNA-seq V2 RSEM values of KL mRNA and beta value for KL methylation were retrieved from The Cancer Genome Atlas HNSCC dataset. Kaplan-Meier survival curves were used to assess the associations of KL expression and methylation with patient survival; multivariate Cox proportional hazards regression models were used to estimate the HRs and their 95\% CIs.

Results: There is a negative relationship between KL gene expression and its promoter DNA methylation in HNSCC. KL gene expression was positively correlated with overall survival, while KL methylation was inversely correlated with the overall survival of HNSCC patients. Furthermore, KL methylation was significantly associated with gender $(P=0.012)$, tumor grade $(P=0.0009)$ and tumor site $(P<0.0001)$. Finally, HNSCC patients with high KL gene expression or low KL DNA methylation had high LC3 but low NSUN2.

Conclusion: KL methylation silenced its gene expression in HNSCC. Low KL expression and high KL methylation can be potential biomarkers for worse prognosis in HNSCC. As the downstream targets, $L C 3$ and NSUN2 could be responsible for the KL expression in HNSCC. Keywords: biomarkers, gene silencing, head neoplasms, neck neoplasms, predictive values, prognosis

\section{Introduction}

Head and neck cancer (HNC) is the sixth most common cancer worldwide, and about $90 \%$ of HNC cases are head and neck squamous cell carcinoma (HNSCC) cases. ${ }^{1}$ Despite the advancements in treatment, the survival of HNSCC patients has remained poor in the past 40 years, with the 5 -year postoperative survival rate being $<50 \%{ }^{2}$ However, if diagnosed at an early stage, the survival rate can be as high as $80 \%{ }^{3}$ Therefore, identification of biomarkers, especially at the early stage, is urgent for improvement of HNCSS patients' survival.

Klotho (KL) is a single-transmembrane protein composed of 1,012 amino acids. ${ }^{4}$ The extracellular domain of KL can be cleaved and released into blood, urine and cerebrospinal fluid, ${ }^{5}$ where it acts as a hormone to regulate physiological processes. ${ }^{6}$ Although it was initially discovered as an antiaging gene in $1997,{ }^{7} \mathrm{KL}$ has recently emerged as a potent tumor suppressor in a wide variety of cancers, including breast cancer, ${ }^{7-9}$ renal cell carcinoma, ${ }^{10}$ thyroid cancer, ${ }^{11}$ lung cancer, ${ }^{12}$ pancreatic cancer, ${ }^{4}$ oral
Correspondence: Ping Wang Department of Pediatrics, Union Hospital, Tongji Medical College, Technology, 1277 jiefangdadao Avenue, Wuhan, Hubei 430022, China

Tel +86 I83 24693797

Fax +86 I83 24693797

Email wang_ping00@yahoo.com 
cancer and esophageal squamous cell carcinoma (ESCC), ${ }^{13}$ whereas in ovarian cancer, its expression was reported to be associated with poor prognosis. ${ }^{14}$ Compared to normal tissues or adjacent tissues, decreased KL gene expression has been consistently detected in many tumors. On the other hand, restoring $\mathrm{KL}$ inhibited cancer cell proliferation. ${ }^{4-8}$ Although studies of KL in HNSCC just started recently and are still very limited, the available data already demonstrated reduced KL levels in oral squamous cell carcinoma (OSCC) and ESCC. ${ }^{13,15} \mathrm{KL}$ expression is epigenetically regulated by its promoter DNA methylation, a DNA modification frequently found in cancers. ${ }^{16} \mathrm{KL}$ promoter hypermethylation has been reported to silence its expression and consequently leads to the loss of its tumor suppressive function. ${ }^{9}$ In fact, KL promoter hypermethylation has been found in malignancies such as HNSCC, ${ }^{17}$ breast cancer, ${ }^{9}$ colorectal cancer ${ }^{18}$ and cervical cancer. ${ }^{19}$ These findings raise the surge of evaluating $\mathrm{KL}$ expression and its DNA methylation as potential prognosticators in HNSCC.

Thus, the purpose of this study was to investigate the significance of KL expression and its DNA methylation in HNSCC survival. To explore the possible underlying mechanisms, we examined their associations with the autophagyassociated gene $L C 3$ as well as the RNA methyltransferase gene NSUN2, a newly discovered prognostic marker for HNSCC. ${ }^{20}$

\section{Materials and methods}

\section{Gene expression, DNA methylation and clinicopathologic data}

The Cancer Genome Atlas HNSCC provisional dataset (www. cbioportal.org) enrolled 530 patients, of whom 522 tumor specimens were analyzed for RNA levels using RNA-seq and for DNA methylation using Illumina DNA methylation array HM450, which were conducted as previously described. ${ }^{21}$ Both upper quartile normalized RNA-seq V2 RNA-seq by expectation maximization (RSEM) values of KL expressions, and beta values of KL, which are the ratios of intensities between methylated and unmethylated alleles to estimate DNA methylation levels, as well as clinicopathologic data were retrieved.

Of the 522 patients who had available data on both RNAseq and DNA methylation of KL, the average age was 60.9 years old (range from 19 to 90). There were 200 patients whose tumor was located at the tongue, 135 had tumor at the pharynx and 186 had tumor at other sites of head and neck. The patients, except for one without indicated gender, were composed of 384 males and 137 females. Based on the information available for race (506 patients), Caucasian patients accounted for $88.1 \%$, African Americans 9.3\%, Asians 2.2\% and Native Americans 0.4\%. One hundred seventeen patients had no smoking history and 391 had it. There were 284 patients with a stage IV disease, 105 patients with stage III, 98 patients with stage II and 20 patients with stage I disease. Sixty-three patients had a tumor with grade I (12.2\%), 304 with grade II (58.8\%) and 150 with grade III (29\%). Post-radiotherapy information was available only for 183 patients, of whom 121 (66.1\%) received radiotherapy after surgery, while 62 did not. The follow-up information was available for 519 patients with the average overall survival being 21.2 months (range from 0.07 to 210.8 months; Table 2).

\section{Statistical analyses}

Statistical analyses were performed using SAS version 9.2 (SAS Institute Inc.). The overall survival in months was calculated as the time from surgery until either the event of death or last follow-up, whichever came first. Spearman correlation was performed to analyze the correlations between KL expression and DNA methylation, NSUN2 or LC3 expression. When KL expression and DNA methylation were treated as categorical variables, they were classified into three groups: low, medium and high, based on the distribution of tertile as the cutoff values. The chi-squared test was used to analyze the associations of KL expression and DNA methylation with clinicopathologic variables. Kaplan-Meier survival curves were used to assess the associations of KL expression and DNA methylation with patient survival, and multivariate Cox proportional hazard regression models were used to estimate the HRs and their 95\% CIs, after adjusting for patients' age at diagnosis, disease stage, tumor grade, gender and smoking status. A $P$-value $<0.05$ was considered statistically significant.

\section{Results \\ Association of $K L$ expression and its DNA methylation with clinicopathologic features in patients}

In the analyzed 522 tumor samples, the average expression level of KL was 11.4 Fragments Per Kilobase of transcript per Million mapped reads (FPKM) ranging from 0 to 1,422 FPKM; the average DNA methylation level was 0.36 ranging from 0.03 to 0.80 (Table 1). A significant negative correlation was observed between KL DNA methylation and its expression. The correlation coefficient was -0.29 (95\% CI: -0.36 to $-0.20, P<0.0001$; Table 1). 
Table 2 shows the associations of KL expression and its DNA methylation with clinicopathologic variables. To limit variation, we first classified patients into three categories: low, medium and high, based on the tertile distribution of KL expression and its methylation, respectively. Although patients with more advanced stage of disease tended to have lower KL expression $(P$-trend $=0.039), \mathrm{KL}$ expression levels between disease stages were not statistically different ( $P=0.183$ ). However, KL expression was found to be significantly associated with tumor sites $(P<0.0001)$. Patients with tumor on tongue tended to have a high KL expression, while tumor on other sites beyond the tongue and pharynx tended to have a low KL expression. No statistically significant associations were found between KL expression with other variables such as tumor grade, race, gender and smoking status.

Table I Spearman correlation between KL expression and DNA methylation

\begin{tabular}{|l|l|l|l|l|l|}
\hline Variable & $\mathbf{n}$ & Mean & SD & Median & Range \\
\hline KL expression & 522 & 23.6 & 71.5 & $I I .4$ & $0-I, 422$ \\
\hline KL DNA methylation & 522 & 0.36 & 0.16 & 0.37 & $0.03-0.80$ \\
\hline $\begin{array}{l}\text { Correlation coefficient } \\
(95 \% \text { Cl) }\end{array}$ & 522 & & -0.29 & \multicolumn{2}{|l|}{$(-0.36,-0.20)$} \\
\hline$P$-value & & & $<0.000 I$ & & \\
\hline
\end{tabular}

KL DNA methylation was found to be significantly associated with gender $(P=0.012)$, tumor grade $(P=0.0009)$ and tumor site $(P<0.0001)$. Female patients tended to have a higher KL methylation than males $(P$-trend $=0.04)$. Patients with higher tumor grade (poor differentiation) tended to have a lower KL methylation, while those with lower tumor grade tended to have a higher KL methylation $(P$-trend $=0.0006)$. In addition, it was found that patients with a disease on tongue tended to have a lower KL methylation, while those with a tumor located beyond the tongue and pharynx tended to have a higher KL methylation $(P$-trend $<0.0001)$. No significant associations were found between $\mathrm{KL}$ methylation with other variables, such as race $(P=0.775)$, smoking status $(P=0.288)$ and disease stage $(P=0.520)$.

\section{Associations of $\mathrm{KL}$ expression and methylation with patient survival}

Kaplan-Meier survival curve analyses stratified by either KL expression or KL methylation levels were first performed to examine the associations of KL expression and DNA methylation with overall survival. The results showed that patients with high KL expression had significantly superior overall survival in comparison to those with low KL expression (log$\operatorname{rank} P=0.002$; Figure 1). The survival curve for patients with

Table 2 Associations of $K L$ expression and DNA methylation with clinicopathologic variables

\begin{tabular}{|c|c|c|c|c|c|c|c|c|c|c|c|}
\hline \multirow[b]{2}{*}{ Variable } & \multirow[b]{2}{*}{$\mathbf{n}$} & \multirow[b]{2}{*}{ Low } & \multicolumn{2}{|c|}{ KL expression } & \multirow[b]{2}{*}{$P$-value } & \multirow[b]{2}{*}{ P-trend } & \multirow[b]{2}{*}{ Low } & \multicolumn{2}{|c|}{ KL methylation } & \multirow[b]{2}{*}{$P$-value } & \multirow[b]{2}{*}{ P-trend } \\
\hline & & & Medium & High & & & & Medium & High & & \\
\hline Gender & 521 & & & & 0.271 & 0.394 & & & & 0.012 & 0.04 \\
\hline Female & 137 & $39(28.5)^{a}$ & $52(38.0)$ & $46(33.6)$ & & & $32(23.4)$ & $56(40.9)$ & $49(35.8)$ & & \\
\hline Male & 384 & $135(35.2)$ & $12 \mid(3 \mid .5)$ & $128(33.3)$ & & & $|4|(36.7)$ & I I8 (30.7) & $125(32.6)$ & & \\
\hline Race & 506 & & & & 0.672 & 0.390 & & & & 0.775 & 0.736 \\
\hline Caucasian & 446 & $146(32.7)$ & $148(33.2)$ & $152(34.1)$ & & & $148(33.2)$ & $152(34.1)$ & $146(32.7)$ & & \\
\hline Other & 60 & $23(38.3)$ & $19(31.7)$ & $18(30.0)$ & & & $20(33.3)$ & $18(30.0)$ & $22(36.7)$ & & \\
\hline Smoking & 508 & & & & 0.282 & 0.270 & & & & 0.288 & 0.115 \\
\hline No & 117 & $37(31.6)$ & $34(29.1)$ & $46(39.2)$ & & & $33(28.2)$ & $39(33.3)$ & $45(38.5)$ & & \\
\hline Yes & 391 & $131(33.5)$ & $136(34.8)$ & $124(31.7)$ & & & $137(35.0)$ & $130(33.3)$ & $124(31.7)$ & & \\
\hline Stage & 507 & & & & 0.183 & 0.039 & & & & 0.520 & 0.141 \\
\hline 1 & 20 & $3(15.0)$ & $8(40.0)$ & $9(45.0)$ & & & $6(30.0)$ & $8(40.0)$ & $6(30.0)$ & & \\
\hline II & 98 & $26(26.5)$ & $32(32.7)$ & $40(40.8)$ & & & $29(29.6)$ & $34(34.7)$ & $35(35.7)$ & & \\
\hline III & 105 & $42(40.0)$ & $31(29.5)$ & $32(30.5)$ & & & $30(28.6)$ & $33(31.4)$ & $42(40.0)$ & & \\
\hline IV & 284 & $97(34.2)$ & $98(34.5)$ & $89(31.3)$ & & & $105(37.0)$ & $93(32.8)$ & $86(30.3)$ & & \\
\hline Grade & 517 & & & & 0.779 & 0.223 & & & & 0.0009 & 0.0006 \\
\hline I & 63 & $24(38.1)$ & $22(34.9)$ & $17(27.0)$ & & & $17(27.0)$ & $16(25.4)$ & $30(47.6)$ & & \\
\hline II & 304 & 101 (33.2) & $102(33.6)$ & $101(33.2)$ & & & $86(28.3)$ & $115(37.8)$ & $103(33.9)$ & & \\
\hline III & 150 & $47(3 \mid .3)$ & $49(32.7)$ & $54(36.0)$ & & & 67 (44.7) & $42(28.0)$ & $4 I(27.3)$ & & \\
\hline Tumor site & 521 & & & & $<0.0001$ & $<0.0001$ & & & & $<0.0001$ & $<0.0001$ \\
\hline Tongue & 200 & $52(26.0)$ & $60(30.0)$ & $88(44.0)$ & & & $79(39.5)$ & $72(36.0)$ & $49(24.5)$ & & \\
\hline Pharynx & 135 & $39(28.9)$ & $52(38.5)$ & $44(32.6)$ & & & $53(39.3)$ & $45(33.3)$ & 37 (27.4) & & \\
\hline Other & 186 & $83(44.6)$ & $61(32.8)$ & $42(22.6)$ & & & $41(22.0)$ & $57(30.7)$ & $88(47.3)$ & & \\
\hline
\end{tabular}

Note: ${ }^{\mathrm{n}} \mathrm{n}(\%)$. 
low KL expression was clearly separated from the curves for those with either medium or high expression. The medians of overall survival were 68.4 months (95\% CI: 42.3-210.8 months) for those with high expression, 56.9 months (95\% CI: 35.8-153.8 months) for those with medium expression and 32.6 months (95\% CI: $22.3-65.8$ months) for those with low expression. The difference in overall survival between those with high and low expression was 25.8 months. The HRs for death were 0.58 (95\% CI: $0.42-0.81)$ for those with high vs low expression and 0.68 (95\% CI: $0.50-0.93)$ for those with medium vs low expression. Conversely, patients with high KL DNA methylation had inferior overall survival in comparison to those with low methylation (log-rank $P=0.057$; Figure 2). The medians of overall survival were 57.9 months (95\% CI: 47.9-159.5 months) for those with low methylation, 48.2 months (95\% CI: 32.5-89.3 months) for those with medium methylation and 37.2 months $(95 \%$ CI: 22.2-65.8 months) for those with high methylation. Patients with low KL DNA methylation had 20.7 months longer overall survival than those with high methylation. The HRs were 1.23 with $95 \%$ CI ranging from 0.87 to 1.74 $(P=0.251)$ for patients with medium vs low methylation and 1.50 with $95 \%$ CI ranging from 1.07 to $2.08(P=0.018)$ for those with high vs low methylation. There was a statistically significant trend $(P$-trend $=0.017)$.

To further validate the Kaplan-Meier survival curve results, we performed multivariate Cox proportional hazard models with the adjustment of potential confounding variables, including patient age at diagnosis, disease stage, tumor grade, gender and smoking status. Again, the multivariate Cox models showed that patients with high KL expression had a lower risk of death than those with low expression (Table 3). The adjusted HRs were 0.69 (95\% CI: 0.50-0.95) for medium vs low $(P=0.025)$ and 0.59 (95\% CI: $0.42-0.83)$ for high vs low $(P=0.003)$. A significantly decreased trend existed $(P=0.002)$. In contrast, high KL DNA methylation significantly increased the risk of death in these patients. The adjusted HRs were 1.24 (95\% CI: 0.86-1.78) for medium vs low methylation $(P=0.248)$ and 1.52 (95\% CI: $1.07-2.17)$ for high vs low methylation $(P=0.019)$. There was a significantly increased trend in the risk of death with increased KL DNA methylation $(P$-trend $=0.018)$.

\section{Associations of $\mathrm{KL}$ expression and its methylation with LC3 and NSUN2}

To explore the possible mechanisms underlying the prognostic factors of KL expression and its DNA methylation in HNSCC survival, we examined their associations with the expression of LC3, an autophagy-related gene, and NSUN2, a tRNA methyltransferase gene, both of which have been shown to be critically involved in HNSCC. KL expression was positively associated with LC3 expression (correlation coefficient $=0.17, P=0.0003$; Figure $3 \mathrm{~A}$ ), whereas $\mathrm{KL}$ methylation was negatively associated with LC3 expression (correlation coef-

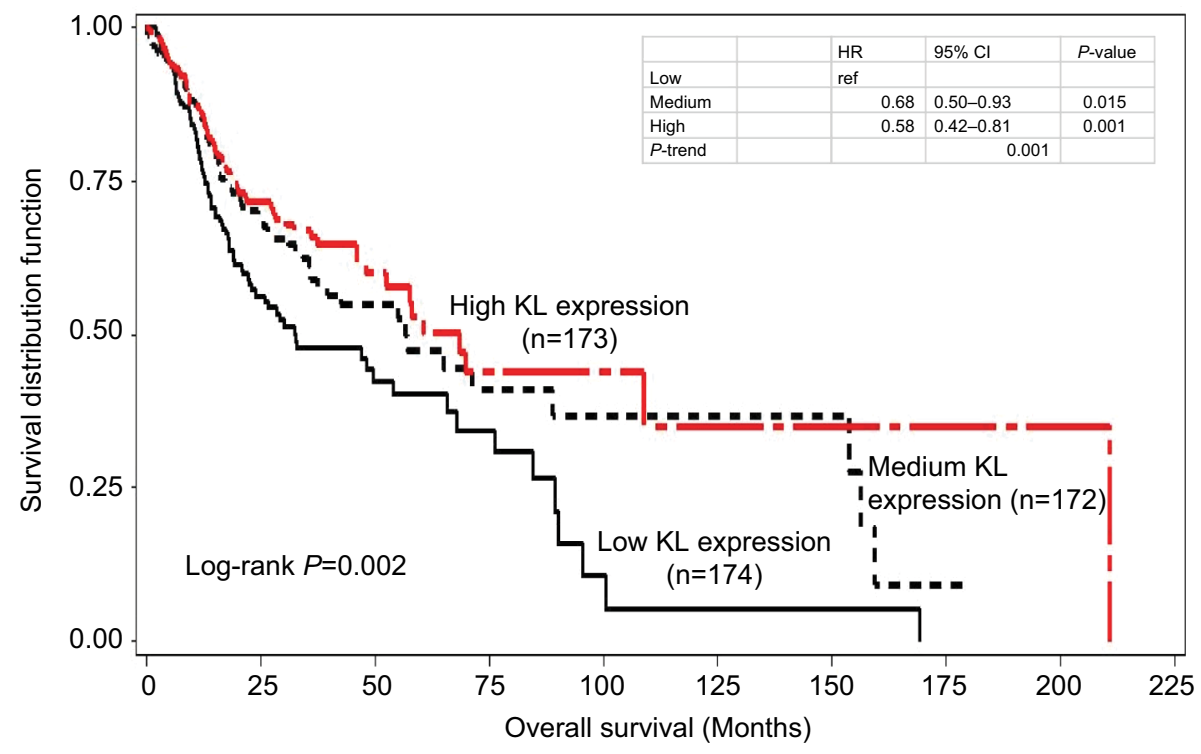

Figure I Kaplan-Meier overall survival curves stratified by KL expression levels.

Notes: In comparison to low expression, high $\mathrm{KL}$ expression had significantly superior overall survival (log-rank $P=0.002$ ). The $\mathrm{HR}$ was 0.58 with $95 \% \mathrm{Cl}$ ranging from 0.42 to $0.8 \mathrm{I}(P=0.00 \mathrm{I})$ for high vs low, while for medium vs low, the $\mathrm{HR}$ was 0.68 with $95 \% \mathrm{Cl}$ ranging from 0.50 to 0.93 ( $P=0.0 \mathrm{I}$ ). There was a statistically significant trend $(P$-trend $=0.00 \mathrm{I})$. 


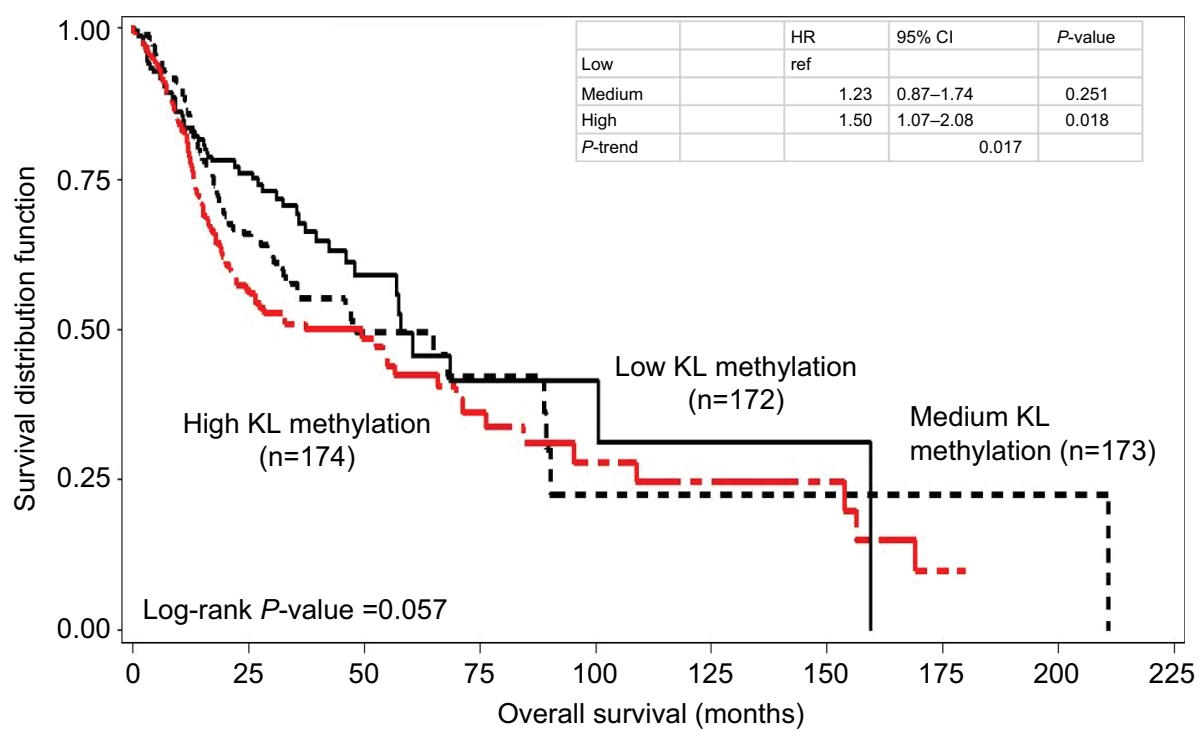

Figure 2 Kaplan-Meier overall survival curves stratified by KL DNA methylation levels.

Notes: High KL DNA methylation had inferior overall survival than low methylation (log-rank $P=0.057$ ). The $\mathrm{HR}$ was $\mathrm{I} .50$ with $95 \% \mathrm{Cl}$ ranging from I.07 to 2.08 ( $P=0.018$ ) for high vs low, while for medium vs low, the $\mathrm{HR}$ was $\mathrm{I} .23$ with $95 \% \mathrm{Cl}$ ranging from 0.87 to $\mathrm{I} .74(P=0.25 \mathrm{I})$. There was a statistically significant trend $(P$-trend $=0.0 \mathrm{I} 7)$.

Table 3 Associations of $\mathrm{KL}$ expression and DNA methylation with the risk of death

\begin{tabular}{|l|l|l|l|}
\hline & & Death & \\
\hline Variable & $\mathbf{H R}^{\mathbf{a}}$ & $\mathbf{9 5 \%} \mathbf{C l}$ & $\boldsymbol{P}$-value \\
\hline KL expression & & & \\
\hline Low & & & \\
\hline Medium & 0.69 & $0.50-0.95$ & 0.025 \\
\hline High & 0.59 & $0.42-0.83$ & 0.003 \\
\hline P-trend & & 0.002 & \\
\hline Age (per 5 years) & $\mathrm{I} .08$ & $\mathrm{I} .0 \mathrm{I}-\mathrm{I} .16$ & 0.020 \\
\hline Stage & $\mathrm{I} .08$ & $0.92-1.26$ & 0.370 \\
\hline Grade & $\mathrm{I} .05$ & $0.85-1.30$ & 0.640 \\
\hline Gender (male vs female) & 0.79 & $0.58-1.08$ & 0.145 \\
\hline Smoking (smoker vs nonsmoker) & $\mathrm{I} .19$ & $0.83-1.69$ & 0.347 \\
\hline KL methylation & & & \\
\hline Low & & & \\
\hline Medium & $\mathrm{I} .24$ & $0.86-1.78$ & 0.248 \\
\hline High & $\mathrm{I} .52$ & $\mathrm{I} .07-2.17$ & 0.019 \\
\hline P-trend & & 0.018 & \\
\hline Age (per 5 years) & $\mathrm{I} .09$ & $\mathrm{I} .02-1.16$ & 0.013 \\
\hline Stage & $\mathrm{I} .1 \mathrm{I}$ & $0.95-1.30$ & 0.202 \\
\hline Grade & $\mathrm{I} .06$ & $0.86-1.3 \mathrm{I}$ & 0.593 \\
\hline Gender (male vs female) & 0.82 & $0.60-1.13$ & 0.223 \\
\hline Smoking (smoker vs nonsmoker) & $\mathrm{I} .20$ & $0.84-1.7 \mathrm{I}$ & 0.313 \\
\hline
\end{tabular}

Note: ${ }^{\mathrm{H} R}$ was estimated in multivariate Cox proportional hazard regression analysis.

ficient $=-0.27, P=1.01 \mathrm{e}-12$; Figure $3 \mathrm{~B})$. On the other hand, $\mathrm{KL}$ expression was found to be negatively associated with NSUN2 expression (correlation coefficient $=-0.16, P=0.0002$; Figure 4A) and KL methylation to be positively related to NSUN2 expression (correlation coefficient $=0.15, P=0.004$; Figure 4B).

\section{Discussion}

To our knowledge, this is the first study to evaluate the prognostic value of KL in predominantly Caucasian HNSCC patients $(88.1 \%)$ with a relatively large sample size (522 available data). We found an obvious negative correlation between KL expression and its DNA methylation, suggesting that KL DNA methylation leads to the silencing of its expression in HNSCC. Consistent with this inverse relationship, we discovered that patients with either high KL expression or low KL DNA methylation had lower risk of death and a superior overall survival, suggesting KL and its DNA methylation can be potential indicators for prognosis in HNSCC. Moreover, this research revealed significant associations of KL expression and its DNA methylation with NSUN2 and LC3, two reported prognosis indicators for HNCSS.

$\mathrm{KL}$ is an antiaging hormone and essentially involved in longevity and cellular homeostasis. It has been reported that $\mathrm{KL}$, as a tumor suppressor, downregulates insulin growth factor (IGF) signaling pathway that is involved in tumorigenesis and progression of many human cancers, including renal cell carcinoma, ${ }^{10}$ breast cancer, ${ }^{22}$ lung cancer,, 923 pancreatic cancer ${ }^{4}$ and HNC., ${ }^{9}, 13,15,24$ Compared to normal tissues, tumor tissues usually have a dramatically reduced KL expression. ${ }^{10,19,25}$ Previous studies have reported reduced KL protein levels in ESCC and OSCC. ${ }^{15,17}$ Furthermore, decreased KL protein levels usually parallel with increased DNA methylation in its promoter region and are associated with poor prognosis for a variety of cancers, such as 
A

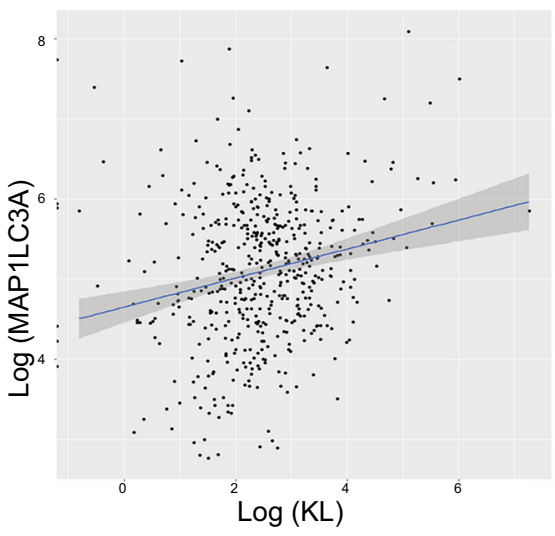

B

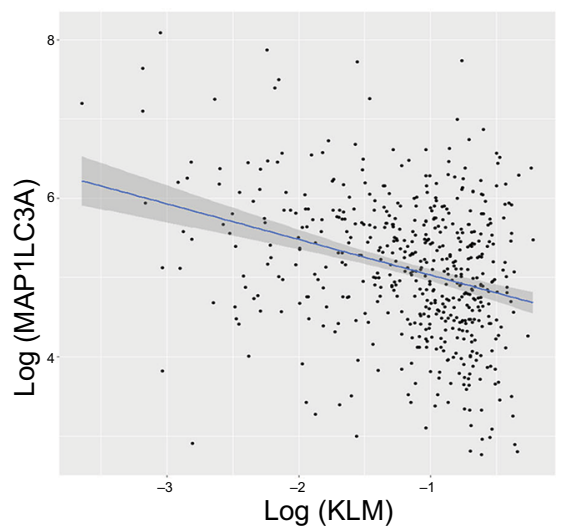

Figure 3 Associations of $K L$ expression and methylation with $L C 3$ gene expression.

Note: Scatter plots and linear regression line $(95 \% \mathrm{Cl}$ in shadow) show that high $\mathrm{KL}$ expression was positively associated with LC3, an autophagy gene (correlation coefficient $=0.17, P=0.0003)(\mathbf{A})$, and that $K L$ methylation was negatively associated with LC3 (correlation coefficient $=-0.27, P=1.0 \mathrm{le}-12)(\mathbf{B})$.

Abbreviations: KL, klotho expression; KLM, klotho methylation.

A

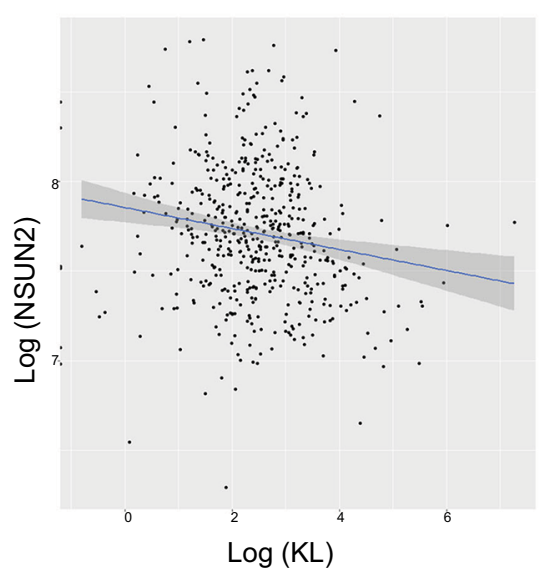

B

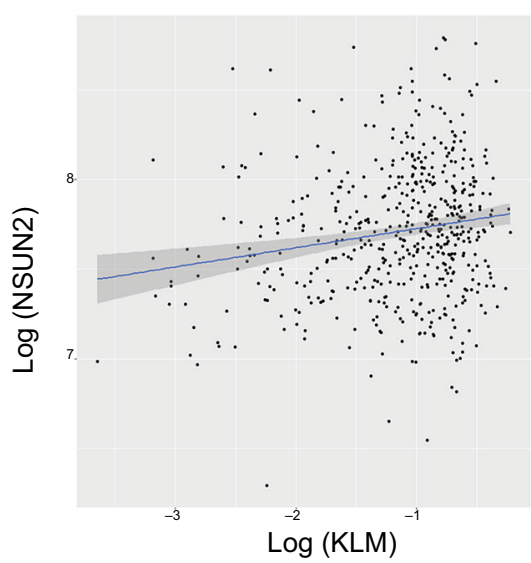

Figure 4 Associations of KL expression and methylation with NSUN2 gene expression.

Note: Scatter plots and linear regression line $(95 \% \mathrm{Cl}$ in shadow) show that high KL expression was negatively associated with NSUN2 (correlation coefficient $=-0.16$, $P=0.0002)(A)$, and that $K L$ methylation was positively associated with NSUN2 (correlation coefficient $=0.15, P=0.004)(B)$.

hepatocellular cancer, ${ }^{24}$ gastric cancer ${ }^{26}$ and ESCC. ${ }^{15}$ In vitro cell line experiments suggest that restoring KL expression or the treatment with soluble KL protein suppresses cancer cell proliferation. ${ }^{19,25}$

In addition to downregulation of IGF signaling, KL may also exert its tumor suppressive function by antagonizing the $\mathrm{Wnt} / \beta$-catenin signaling pathway, ${ }^{27,28}$ thereby leading to the suppression of oncogenes, such as c-myc and cyclin D1, which are abnormally activated in tumors. Furthermore, downregulation of the fibroblast growth factor pathwaymediated phosphorylation of Akt and ERK1/2 has been attributed to the tumor suppressive effect of KL in pancreatic cancer. ${ }^{4} \mathrm{KL}$ has also been reported to inhibit TGF- $\beta 1$-induced epithelial-mesenchymal transition (EMT) by directly bind- ing to TGF- $\beta$ receptor. ${ }^{29}$ Our previous study revealed that high expression of TWIST1, a key regulator in EMT, was associated with poor survival in HNSCC..$^{30}$ In this study, we observed a significantly positive relationship between $\mathrm{KL}$ mRNA levels and the overall survival of HNSCC patients. For the patients with high KL expression, the overall survival is generally 25.8 months longer than in patients with low $\mathrm{KL}$ expression. This finding adds more evidence to support $\mathrm{KL}$ as a tumor suppressor in the context of human cancers.

Due to the enriched $\mathrm{CpG}$ sites (aka CpG island) in the promoter region, $\mathrm{KL}$ expression can be epigenetically silenced by DNA methylation, ${ }^{9,25}$ and the demethylation agent 5 -aza2'-deoxycytidine restores KL expression. ${ }^{26,31}$ As expected, in this study, we found a significantly negative correlation 
between KL DNA methylation and expression in HNSCC tissues. Consistently, we also observed that the correlation of KL methylation with disease survival was always inversely parallel to that of KL expression. That is, lower KL methylation was associated with lower risk of death and superior overall survival. These findings are consistent with previous reports showing that tumor tissues usually had higher KL methylation compared to adjacent normal tissues, ${ }^{9,18,26}$ and that high KL methylation was associated with poor prognosis and severity of several human cancers. ${ }^{18,24,32}$ For example, Rubinek et al reported that breast cancer patients with high KL methylation had poor prognosis. ${ }^{9}$ A similar result of high KL methylation and poor prognosis was reported in hepatocellular carcinoma. ${ }^{24}$ In tumor tissues from OSCC patients, DNA methyltransferase $3 \mathrm{a}$ was found to be higher and the KL protein level was lower than in normal tissues. ${ }^{13}$ Together, these data suggest that DNA methylation of KL plays an important role in the progression of HNSCC. ${ }^{33,34}$ However, a research done in HNSCC samples from Saudi Arabia reported that in HNSCC patients with moderately differentiated tumors, KL methylation was related to better survival rate, compared to that in those without KL methylation. ${ }^{17}$

Autophagy is critical for maintaining cellular homeostasis by the degradation of misfolded proteins and damaged organelles. In cancers, autophagy can play dual roles as tumor promotor or suppressor depends on the tumor stages. At the early stage, autophagy-mediated cell survival may contribute to cancer etiology and progression, while at a later stage, activation of autophagy can be antitumorigenic. ${ }^{35}$ In oral tongue squamous cell carcinoma (OTSCC) patients, there is evidence that the expression of autophagy-related gene beclin-1 was decreased in the tumor tissue and correlated with poor prognosis. In addition, overexpression of this autophagy gene inhibited proliferation and clonogenicity of OTSCC cells. ${ }^{36}$ LC3 is a specific marker for the autophagosome. In this study, we found that either high KL expression or low KL DNA methylation correlated positively with LC3 level in HNSCC. KL has been reported to exert its tumor suppressive function by activation of autophagy in several cancers. ${ }^{37,38}$ Our data thus suggest a link between LC3 and KL in HNSCC.

NSUN2 is an RNA methyltransferase involved in cell proliferation and differentiation. ${ }^{39,40}$ NSUN2 is a direct target of $\mathrm{MYC},{ }^{41}$ and its overexpression has been reported to contribute to the development of several cancers, such as breast cancer $^{42}$ and HNSCC. ${ }^{20}$ Moreover, high NSUN2 level is positively associated with poor prognosis in HNSCC. ${ }^{20}$ Recently, it was discovered that NSUN2 is also a transcription target of the Lef1/ $\beta$-catenin complex, ${ }^{39}$ a well-known downstream pathway inhibited by KL. ${ }^{43}$ In agreement with these reports, we found that NSUN2 was negatively correlated with KL expression but positively correlated with $\mathrm{KL}$ methylation in HNSCC. To our knowledge, this is the first study to demonstrate a relationship between KL and NSUN2 in HNSCC.

\section{Conclusion}

This study points out that KL gene expression and its promoter DNA methylation are potential prognostic markers for HNSCCs. Moreover, our findings also suggest correlations of KL with both LC3 and NSUN2 in HNSCC. Further studies on the roles of LC3 and NSUN2 as downstream targets regulated by KL in HNSCC are warranted.

\section{Acknowledgments}

The results shown in this manuscript are based upon the data generated by The Cancer Genome Atlas Research Network http://cancergenome.nih.gov/. This work was funded by the National Natural Science Foundation of China (grant number 81600805).

\section{Author contributions}

PW designed the study. YZ, XC and QC collected and analyzed the TCGA data. YZ, LW and XZ wrote the manuscript. All authors contributed to data analysis, drafting or revising the article, gave final approval of the version to be published, and agree to be accountable for all aspects of the work.

\section{Disclosure}

The authors report no conflicts of interest in this work.

\section{References}

1. Ferlay J, Soerjomataram I, Dikshit R, et al. Cancer incidence and mortality worldwide: sources, methods and major patterns in GLOBOCAN 2012. Int J Cancer. 2015;136(5):E359-E386.

2. Braakhuis BJ, Leemans CR, Visser O. Incidence and survival trends of head and neck squamous cell carcinoma in the Netherlands between 1989 and 2011. Oral Oncol. 2014;50(7):670-675.

3. Lefebvre JL. Current clinical outcomes demand new treatment options for SCCHN. Ann Oncol. 2005;16(suppl_6):vi7-vi12.

4. Abramovitz L, Rubinek T, Ligumsky H, et al. KL1 internal repeat mediates Klotho tumor suppressor activities and inhibits bFGF and IGF-I signaling in pancreatic cancer. Clin Cancer Res. 2011;17(13):4254-4266.

5. Imura A, Iwano A, Tohyama O, et al. Secreted Klotho protein in sera and CSF: implication for post-translational cleavage in release of Klotho protein from cell membrane. FEBS Lett. 2004;565(1-3):143-147.

6. Takahashi Y, Kuro-O M, Ishikawa F. Aging mechanisms. Proc Natl Acad Sci U S A. 2000;97(23):12407-12408.

7. Kuro-O M, Matsumura Y, Aizawa H, et al. Mutation of the mouse Klotho gene leads to a syndrome resembling ageing. Nature. 1997;390(6655):45-51.

8. Wolf I, Levanon-Cohen S, Bose S, et al. Klotho: a tumor suppressor and a modulator of the IGF-1 and FGF pathways in human breast cancer. Oncogene. 2008;27(56):7094-7105. 
9. Rubinek T, Shulman M, Israeli S, et al. Epigenetic silencing of the tumor suppressor Klotho in human breast cancer. Breast Cancer Res Treat. 2012;133(2):649-657.

10. Gigante M, Lucarelli G, Divella C, et al. Soluble serum $\alpha$ Klotho is a potential predictive marker of disease progression in clear cell renal cell carcinoma. Medicine. 2015;94(45):e1917.

11. Dai D, Wang Q, Li X, Liu J, Ma X, Xu W. Klotho inhibits human follicular thyroid cancer cell growth and promotes apoptosis through regulation of the expression of stanniocalcin-1. Oncol Rep. 2016;35(1):552-558.

12. Usuda J, Ichinose S, Ishizumi T, et al. Klotho predicts good clinical outcome in patients with limited-disease small cell lung cancer who received surgery. Lung Cancer. 2011;74(2):332-337.

13. Adhikari BR, Uehara O, Matsuoka $\mathrm{H}$, et al. Immunohistochemical evaluation of Klotho and DNA methyltransferase 3a in oral squamous cell carcinomas. Med Mol Morphol. 2017;50(3):155-160.

14. Lu L, Katsaros D, Wiley A, de la Longrais IA, Puopolo M, Yu H. Klotho expression in epithelial ovarian cancer and its association with insulin-like growth factors and disease progression. Cancer Invest. 2008;26(2):185-192.

15. Tang X, Fan Z, Wang Y, et al. Expression of Klotho and $\beta$-catenin in esophageal squamous cell carcinoma, and their clinicopathological and prognostic significance. Dis Esophagus. 2016;29(3):207-214.

16. Schuebel KE, Chen W, Cope L, et al. Comparing the DNA hypermethylome with gene mutations in human colorectal cancer. PLoS Genet. 2007;3(9):1709-1723.

17. Alsofyani AA, Alsiary RA, Samkari A, et al. Prognostic potential of KLOTHO and SFRP1 promoter methylation in head and neck squamous cell carcinoma. J Appl Genet. 2017;58(4):459-465.

18. Perveez M,Ajaz M, Afroze D. Promoter hypermethylation of KLOTHO; an anti-senescence related gene in colorectal cancer patients of Kashmir valley. Mol Biol Res Commun. 2015;4(4):217-224.

19. Lee J, Jeong DJ, Kim J, et al. The anti-aging gene KLOTHO is a novel target for epigenetic silencing in human cervical carcinoma. Mol Cancer. 2010;9(1):109.

20. Lu L, Zhu G, Zeng H, Xu Q, Holzmann K. High tRNA transferase NSUN2 gene expression is associated with poor prognosis in head and neck squamous carcinoma. Cancer Invest. 2018;36(4):246-253.

21. Cancer Genome Atlas Research Network. Comprehensive molecular characterization of gastric adenocarcinoma. Nature. 2014;513(7517):202-209.

22. Ligumsky H, Rubinek T, Merenbakh-Lamin K, et al. Tumor suppressor activity of Klotho in breast cancer is revealed by structure-function analysis. Mol Cancer Res. 2015;13(10):1398-1407.

23. Wang Y, Chen L, Huang G, et al. Klotho sensitizes human lung cancer cell line to cisplatin via PI3k/Akt pathway. PLoS One. 2013;8(2):e57391.

24. Xie B, Zhou J, Yuan L, et al. Epigenetic silencing of Klotho expression correlates with poor prognosis of human hepatocellular carcinoma. Hum Pathol. 2013;44(5):795-801.

25. Pan J, Zhong J, Gan LH, et al. Klotho, an anti-senescence related gene, is frequently inactivated through promoter hypermethylation in colorectal cancer. Tumour Biol. 2011;32(4):729-735.
26. Wang L, Wang X, Wang X, et al. Klotho is silenced through promoter hypermethylation in gastric cancer. Am J Cancer Res. 2011;1(1): 111-119.

27. Kurosu H, Yamamoto M, Clark JD, et al. Suppression of aging in mice by the hormone Klotho. Science. 2005;309(5742):1829-1833.

28. Liu H, Fergusson MM, Castilho RM, et al. Augmented Wnt signaling in a mammalian model of accelerated aging. Science. 2007; 317(5839):803-806.

29. Doi S, Zou Y, Togao O, et al. Klotho inhibits transforming growth factor-beta1 (TGF-beta1) signaling and suppresses renal fibrosis and cancer metastasis in mice. J Biol Chem. 2011;286(10):8655-8665.

30. Zhu Y, Zhang W, Wang P. Smoking and gender modify the effect of TWIST on patient survival in head and neck squamous carcinoma. Oncotarget. 2017;8(49):85816-85827.

31. Tsai KD, Lee WX, Chen W, et al. Upregulation of PRMT6 by LPS suppresses Klotho expression through interaction with NF- $\kappa \mathrm{B}$ in glomerular mesangial cells. J Cell Biochem. 2018;119(4):3404-3416.

32. Yang W, Wang X, Li X, et al. The specific methylation characteristics of cancer related genes in Chinese colorectal cancer patients. Tumour Biol. 2014;35(8):8267-8279.

33. Demokan S, Dalay N. Role of DNA methylation in head and neck cancer. Clin Epigenetics. 2011;2(2):123-150.

34. Worsham MJ, Stephen JK, Chen KM, et al. Delineating an epigenetic continuum in head and neck cancer. Cancer Lett. 2014;342(2):178-184.

35. Sannigrahi MK, Singh V, Sharma R, Panda NK, Khullar M. Role of autophagy in head and neck cancer and therapeutic resistance. Oral Dis. 2015;21(3):283-291.

36. $\mathrm{Hu} \mathrm{Z}$, Zhong $\mathrm{Z}$, Huang $\mathrm{S}$, et al. Decreased expression of Beclin-1 is significantly associated with a poor prognosis in oral tongue squamous cell carcinoma. Mol Med Rep. 2016;14(2):1567-1573.

37. Shu G, Xie B, Ren F, et al. Restoration of Klotho expression induces apoptosis and autophagy in hepatocellular carcinoma cells. Cell Oncol. 2013;36(2):121-129.

38. Xie B, Zhou J, Shu G, et al. Restoration of Klotho gene expression induces apoptosis and autophagy in gastric cancer cells: tumor suppressive role of Klotho in gastric cancer. Cancer Cell Int. 2013;13(1):18.

39. Blanco S, Kurowski A, Nichols J, Watt FM, Benitah SA, Frye M. The RNA-methyltransferase Misu (NSun2) poises epidermal stem cells to differentiate. PLoS Genet. 2011;7(12):e1002403.

40. Hussain S, Benavente SB, Nascimento E, et al. The nucleolar RNA methyltransferase Misu (NSun2) is required for mitotic spindle stability. $J$ Cell Biol. 2009;186(1):27-40.

41. Frye M, Watt FM. The RNA methyltransferase Misu (NSun2) mediates Myc-induced proliferation and is upregulated in tumors. Curr Biol. 2006;16(10):971-981.

42. Frye M, Dragoni I, Chin SF, et al. Genomic gain of 5 p15 leads to overexpression of Misu (NSUN2) in breast cancer. Cancer Lett. 2010;289(1): 71-80.

43. Chang B, Kim J, Jeong D, et al. Klotho inhibits the capacity of cell migration and invasion in cervical cancer. Oncol Rep. 2012;28(3):1022-1028.
Cancer Management and Research

\section{Publish your work in this journal}

Cancer Management and Research is an international, peer-reviewed open access journal focusing on cancer research and the optimal use of preventative and integrated treatment interventions to achieve improved outcomes, enhanced survival and quality of life for the cancer patient. The manuscript management system is completely online and includes

\section{Dovepress}

a very quick and fair peer-review system, which is all easy to use. Visit $\mathrm{http}: / / \mathrm{www}$.dovepress.com/testimonials.php to read real quotes from published authors. 